\title{
"Como si fueran varones": discurso y performance de género en espacios públicos como conflicto generacional
}

\author{
"Como si fueran varones": gender discourse and \\ performance in public spaces as a generational conflict
}

\author{
DRa. Verónica FILARdo* \\ Grupo de estudios Urbanos y Generacionales, \\ Departamento de Sociología \\ Facultad de Ciencias Sociales, Universidad de la República \\ ORCID: 0000-0003-2304-069X
}

MG. JimENA PANDOLFi*

Grupo de estudios Urbanos y Generacionales, Departamento de Sociología

Facultad de Ciencias Sociales, Universidad de la República ORCID: 0000-0002-2906-2649

Recibido: 20/07/2019

Aceptado: 03/09/2020

doi: https://doi.org/10.20318/femeris.2020.5761

\begin{abstract}
Resumen. Las performances de género son la expresión dramatúrgica de las identidades de género; son acciones que en el espacio público muestran para "otros" el ajuste o desvío de lo que representa ser varón, mujer, trans ${ }^{* * *}$. El trabajo enfoca en los conflictos sociales - mínimos, opacos- que se identifican a través del análisis de habla (Canales, 2013) de un fragmento de un grupo de discusión realizado en el marco del proyecto Jóvenes, ciudades y generaciones (CSIC; 2014-2019). El análisis revela repertorios de acción posibles según aquello que se identifica como el género de las personas que los performan, indicando tensiones y disputas entre generaciones de mujeres (nacidas entre 1990-2000 y nacidas antes de 1960). Se pone en evidencia así, diferentes mandatos de género -discursos que señalan qué debe hacerse, cómo, cuándo, dónde, quién(es) y con quién(es) en los espacios públicos de la ciudad-. Lo que está en juego
\end{abstract}

\footnotetext{
*veronica.filardo@cienciassociales.edu.uy

**jimena.pandolfi@cienciassociales.edu.uy

*** “Varón”, "mujer", "trans" aluden a distintas expresiones de identidad de género. Se utilizan para referir a categorías cultural y socialmente situadas a las que se les atribuye un conjunto de atributos identificados como "masculinos" y "femeninos" a partir de sistemas sexo-genéricos. El término "trans" es una categoría amplia utilizada para describir distintas formas de transgresión de expresiones de género hegemónicas. El denominador común de estas formas es que el sexo asignado al nacer no concuerda con las expectativas de comportamiento e identificación de género que predominan un momento histórico determinado. A modo de ejemplo, "trans" engloba categorías identitarias como "travesti", "transexual" o "transgénero".
} 
-cómo deben comportarse las mujeres en el espacio público urbano- manifiesta la lucha de poder en torno a ideologías de género, que enfrenta a generaciones (mujeres mayores y mujeres jóvenes) en Uruguay.

Palabras clave: performance de género, generaciones, espacios públicos, conflictos generacionales, análisis de discurso.

Abstract. Gender performances are the dramaturgical expression of gender identities; they are actions that show for "others" the adjustment or deviation of what it means to be men, women, trans. The work focuses on social conflicts - minimal, opaque - that are identified through the "speech analysis" (Canales, 2013) of a fragment of a discussion group carried out within the research "Youth, Cities and Generations" (CSIC; 2014-2019). The analysis reveals possible repertoires of action according to what is identified as the gender of the people who perform them, indicating tensions and disputes between generations of women (born between 1990-2000 and born before 1960). Discourses that indicate what should be done, how, when, where, who and with whom in public spaces, are different between generations of women (born between 1990 and 2000 and born before 1960), evidencing different gender dictations, tensions, and conflicts. What is in dispute - how women should behave in the urban public space - manifests the power struggle over gender ideologies, which confronts generations (older women and young women) in Uruguay.

Keywords: gender performances, generations, public spaces, generational conflicts, speech analysis.

\section{Introducción}

Reflexionar sobre sexualidad y las relaciones de género desde la sociología implica reconocer el carácter histórico y cultural de las construcciones identitarias, las emociones y repertorios de acción asociados. Los comportamientos vinculados a los sistemas sexo-genéricos no son universales o con significados aislados, por el contrario, éstos se configuran en un entramado complejo de prácticas y relaciones sociales de reproducción y cambio. De este modo, nociones como placer, deseo, identidades sexuales se van edificando a partir de la delimitación de las prohibiciones y los espacios para su transgresión. Se trata de ideologías, normativas sobre la sexualidad que poseen rasgos persistentes, así como de innovación en un determinado contexto socio-histórico.

En el Uruguay de la década iniciada en los 2000, temáticas relacionadas a los derechos sexuales y reproductivos, la diversidad sexual y las relaciones de género han sido colocadas en la agenda pública y, en algunas ocasiones se han traducido en iniciativas, acciones o normativas. Más aún, en las últimas décadas se ha observado un aumento constante, aunque lento y paulatino, de la participación de las mujeres en el mercado de trabajo, cambios en la composición de los hogares, el aporte de varones y mujeres en los ingresos del hogar (SIG-Inmujeres 2016), entre otros, que dan la pauta de transformaciones en el campo de los vínculos, relaciones y estructuración de los comportamientos en base a identidades sexuales como el género.

La persistencia de rasgos de ideologías heteronormativas históricas sobre la sexualidad coexistiendo con otros de carácter innovador, a la vez que habilitan la emergencia de debates, reconocimiento de nuevos derechos sexuales, transformación de repertorios de acción y ejercicio de la sexualidad, implican la emergencia de tensiones y conflictos, dis- 
putas de poder en este campo. El modo en que los mismos se expresan atraviesa diversas áreas de la vida social en un entramado complejo de coexistencia de múltiples significados, ideologías y representaciones sobre las identidades de género.

Interesa, en este contexto, reflexionar sobre los conflictos que se expresan en un campo específico de la vida social: las performances de género en los espacios públicos urbanos $^{1}$. Hablaremos aquí de performances de género con el objetivo de denominar la expresión dramatúrgica de las identidades de género, es decir, aquellas acciones que expresan de modo significante para "otros" determinadas representaciones sobre las mismas. En este marco, el objetivo del presente trabajo será analizar los discursos sobre dichas performances que expresan mandatos de género, es decir, aquellos discursos que señalan qué debe hacerse, cómo debe hacerse, cuándo, dónde, quién(es) y con quién(es) en los espacios públicos y, en particular, cómo estos discursos establecen repertorios de acción posibles según aquello que se identifica como el género de las personas que los performan. A modo de hipótesis orientadora se sostendrá que existe una disputa de poder sobre las ideologías de género, observable a partir de dichos discursos, que, a la vez que evidencia el carácter performativo del género, se expresa como conflicto generacional.

Este artículo es parte de un trabajo de investigación más amplio realizado a partir de veintiún grupos de discusión con habitantes de las ciudades de Montevideo, Salto y Maldonado $^{2}$. Se identificaron en los discursos de los participantes adultos y adultos mayores, aquellas alusiones que expresan sobreentendidos y las lógicas argumentativas sobre cómo las personas se deben comportar y vincular, fundamentalmente según su género, en los espacios públicos. Se analiza quiénes emiten los discursos, quiénes son objeto de los mismos, qué se expresa y de qué modo. Este trabajo persigue este objetivo, pero se concentra en el análisis de un fragmento de un grupo de discusión. Para ello, se adoptará la propuesta de análisis de discurso de Canales (2013). Es decir, se atiende a los efectos sígnicos y semiotizados de sentido para, en una segunda instancia, preguntar por la estructura y los procesos sociales.

En el primer apartado se presenta esquemáticamente el tipo de análisis a realizar; en el segundo se realizan algunas consideraciones metodológicas para la correcta inter-

\footnotetext{
${ }^{1}$ La idea de "espacio público" posee múltiples acepciones. Algunas referencias clásicas aluden a su sentido político, a su articulación con la "esfera pública". Desde esta perspectiva, autores con puntos de vista diversos, como Habermas, Arendt, Bobbio, Honneth o Frazer, lo han abordado así en términos de poder, derecho, democracia, reconocimiento, y/o construcción de ciudadanía (Filardo et al, 2005). En este trabajo utilizamos el concepto para referir a los espacios públicos de la ciudad. Los debates en torno a qué refiere esta categoría entre los estudios urbanos también es de larga data. Es de particular interés aquí la referencia a la perspectiva del habitante sobre estos espacios La misma se caracteriza como una mirada "laxa", independiente de las categorías normativas y asociada a espacios de encuentros con otros (Filardo et al, 2005). Es en este sentido que se adopta la categoría en este trabajo. Se trata de espacios públicos urbanos (calles, parques, plazas, ferias, playas, etc.) teóricamente de acceso libre y sin restricciones a los ciudadanos.

${ }^{2}$ A partir de los grupos de discusión realizados en el marco del Proyecto CSIC Grupos I+D del "Grupo de Estudios Urbanos y Generacionales" de nombre "Jóvenes, ciudad y generaciones", de los cuales nueve grupos fueron realizados en la ciudad de Montevideo definidos en base a tres grupos de edad (jóvenes de 18 a 29 años, adultos de 30 a 60 años, y adultos mayores de 61 años) y tres niveles socioeconómicos (bajo, medio alto) según el Índice de Nivel Socioeconómico (2011). En las ciudades de Sato y Maldonado se realizaron seis grupos en cada una utilizando los mismos tramos de edad y cortes según nivel socioeconómico (medio-bajo y medio-alto). Los grupos fueron realizados entre setiembre y diciembre del 2016. El fragmento a partir del que se trabaja en este artículo aplicando el análisis sociológico del habla propuesto por Canales (2013) corresponde a un grupo de mayores de 60 años, en la ciudad de Maldonado durante el año 2016.
} 
pretación de resultados. En el tercer apartado se propone el análisis a partir de la selección de una cita proveniente del material empírico, con el objetivo de descomponer sus elementos y discernir las lógicas argumentativas que describen los cambios generacionales percibidos por los adultos en los comportamientos de varones y mujeres jóvenes en los espacios públicos ${ }^{3}$. Los siguientes cuatro apartados presentan las principales líneas de análisis que habilita este estudio. Finalmente, se presentan algunas reflexiones finales a modo de conclusión.

\section{Analizar el habla desde la sociología}

Manuel Canales (2013) propone la existencia de una profunda convergencia entre las sociologías comprensivas y la semiótica del texto. Mientras que la primera parte del sentido trata de comprender a los sujetos en sociedad, la segunda llega al sentido al explicar cómo se genera la comprensión de un texto. Bajo este entendido, señala, el análisis de sentido del que hablan ambas tradiciones es uno y el mismo, a la vez que observable tanto como semiosis textual como significado social.

(...) el sentido de los textos es también el sentido con que los sujetos entienden y se entienden como tales. Por otra parte, para las ciencias sociales del sentido, queda asumir que nuestro objeto/sujeto de estudio pertenece, precisamente por existir en el registro del sentido, al orden del hablar, de significar con palabras (Canales 2013: 174).

Desde esta perspectiva, Canales propone una práctica para el análisis del discurso basada en tres tradiciones teóricas que habilitan articular diversos niveles de comprensión e interpretación de la realidad social. En primer lugar, a partir del análisis semiológico de textos se "disciplina la escucha," es decir, se atiende a los efectos sígnicos en que se forman los efectos de sentido. En segundo lugar, y al mismo tiempo que se realiza el análisis descrito en el primer punto, se retoma la sociología comprensiva para reconstruir los efectos semiotizados de sentido, es decir, los modos en que el sujeto conoce su mundo

\footnotetext{
${ }^{3}$ Emerge con frecuencia en los grupos de discusión, un relativo consenso entre los adultos y los adultos mayores respecto a la relevancia de un conjunto de transformaciones y cambios que identifican como generacionales. Estos tienen que ver con las relaciones de género y, en particular, se refieren al modo en que varones y mujeres jóvenes se comportan y cuáles son sus repertorios de acción en los espacios públicos. La cita analizada fue seleccionada a partir de un conjunto, que han sido agrupadas bajo el mismo código. Sin ánimo de exhaustividad, algunas de ellas que provienen de diferentes grupos son:

"Hoy las chiquilinas están sentadas en la vereda, conversando, fumando, dialogando, hacen campamento. Antes teníamos otra visión, cuando una chiquilina andaba caminando con un chiquilín pensábamos ya que eran novios, ahora no, anda con aquel y no son novios." (Montevideo, mujer adulta mayor, NSE medio)

"A mí, te digo una cosa sinceramente, que me pasa con la juventud. Yo fui criada de una manera, en otra época y hay muchas cosas que yo me cuesta horrores aceptar de hoy en día. Por ejemplo, ver chiquilinas de quince, dieciséis años, con un cigarrillo en la mano a las carcajadas por la calle" (Salto, mujer adulta, NSE medio-alto)

"Incluso la libertad de poder pasear por (la calle) 18 (de julio) con una pareja del mismo sexo. Yo los veo y realmente digo 'cuando yo era gurisa para mí era impensable' porque hasta con un varoncito te escondías para darte un beso". (Montevideo, mujer adulta, NSE medio)
} 
y a sí mismo. Finalmente, en tercer lugar, propone pasar de la comprensión a la interpretación, "escuchar la escucha anterior" para preguntar por la estructura y los procesos sociales. De este modo, no solo se atiende a los efectos semióticos sino a las "situaciones existenciales del hablante," dado que "no se habla igual el mismo dicho según sea la posición del hablante" (Canales 2013: 177). Se trata, de este modo, de identificar la existencia de conflictos y tensiones intrasubjetivas e intersubjetivas que dan cuenta de fracturas y tensiones del orden social.

\section{Consideraciones metodológicas}

El análisis de los grupos constituidos por adultos y adultos mayores, permitió detectar algunas regularidades que se interpretan como un conflicto latente entre mujeres nacidas en el periodo 1990-2000 y aquellas nacidas antes de 1960, aunque son expresadas como diferencias entre "mujeres jóvenes" y "mujeres adultas o adultas-mayores". Estos hallazgos remiten a diferentes dimensiones: sobre el significado atribuido al ser mujer (Filardo, 2018) sobre las posibilidades de estudiar, a partir de un abanico considerado muy amplio de opciones y de menores restricciones para el acceso que antes; sobre el uso de las nuevas tecnologías que estructuran la sociedad actual y que hace que los de mayor edad se sientan "relegados", sobre las condiciones socio-históricas que permiten una mayor libertad de los jóvenes en todos los planos, y sobre los comportamientos de los jóvenes (en particular de las jóvenes) en los espacios públicos urbanos. Estas tensiones que se expresan como luchas generacionales, son presentadas en otro trabajo que titulamos Narrativas de la pérdida (Filardo, 2020) y que interpreta los discursos producidos por los grupos de discusión de adultos y adultos mayores, como la manifestación de una doble pérdida: en primer lugar que cuando fueron jóvenes no pudieron hacer -aunque hubieran querido- los que los jóvenes hoy sí pueden (y hacen); y en segundo lugar que ya no son jóvenes. Este trabajo enfoca en una de esas dimensiones del conflicto y de la pérdida: el comportamiento de las mujeres jóvenes en el espacio público de la ciudad. Para ello, haremos uso de la propuesta de Canales (2013), cercana al análisis narrativo que proponen Riessman (2001), Bruner (1991) y Polkinghorne (1998), en el entendido que este abordaje permite enlazar biografías, posiciones sociales e historia:

"Como dijo Mills hace mucho tiempo, lo que llamamos 'problemas personales' se ubican en tiempos y lugares particulares, y las narrativas de los individuos sobre sus problemas son obras de la historia, tanto como lo son de los individuos, los espacios sociales que habitan y las sociedades en las que viven. El análisis de las narrativas personales puede iluminar "la acción y los significados individuales y colectivos, tanto como los procesos sociales mediante los cuales la vida social y las relaciones humanas se construyen y cambian" (Laslett 1999: 392)". (Riessman, 2001:697) ${ }^{4}$

\footnotetext{
${ }^{4}$ Traducción propia de "As Mills said long ago, what we call "personal troubles" are located in particular times and places, and individuals' narratives about their troubles are works of history, as much as they are about individuals, the 
Conocer y explicitar lo que provoca el surgimiento de un determinado discurso en un grupo de discusión es clave para la interpretación de lo que este hecho hace emerger. Las consignas del trabajo de los grupos de discusión de los que se toma el material empírico, versaban sobre clases de edad y cuidad. Los disparadores que hacen emerger discursos que expresan mandatos vinculados a las identidades de género y el espacio público, suelen ser las respuestas a las preguntas sobre las diferencias que se identifican entre generaciones. A modo de ejemplo, frente a la pregunta “ $¿ E s$ diferente ser joven hoy que cuando ustedes eran jóvenes?" surgen un conjunto de ideas asociadas a cómo varones y mujeres jóvenes se comportan "en la calle," "cuando los ves en la vereda" entre otras referencias asociadas estos espacios.

\section{Performance de género y generaciones}

A continuación se desarrolla el análisis a partir de la selección de una cita proveniente del material empírico utilizado en el presente trabajo con el objetivo de descomponer sus elementos y discernir las lógicas argumentativas que describen los cambios generacionales percibidos por los adultos en los comportamientos de varones y mujeres jóvenes en los espacios públicos. El segmento proviene de un grupo de adultos de 30 a 59 años de nivel socioeconómico bajo realizado en la ciudad de Maldonado, se trata de la respuesta de una participante mujer frente a la pregunta del moderador respecto a si los jóvenes de ahora son distintos de los jóvenes de antes. El segmento seleccionado es el siguiente:

A mí lo que me choca, a mi lo que me choca entre los jóvenes, ya los estoy viendo desde los 13 años, no lo estoy mirando ni siquiera desde los 15, 16, lo que me molesta, porque uno no lo vivió eso, y si tenía ganas no lo podía hacer porque tenía otra enseñanza, es que uno ve las chicas que quieren comparase a los varones. Andan con... se visten o se peinan igual, andan con la botella de cerveza con 14, 15 años por las calles. Y que puede tomar la chica, vamo' a decir, somos todos iguales, tenemos los mismos derechos pero yo me opongo que una chica, una niña, una chiquilina, una adolescente, ande con una botella como si fuera un hombre. Entonces, a veces eso me choca un poco la cabeza, como que las mujeres, digamos, las niñas o las jóvenes quieren comparase un poco con el mismo derecho del hombre. 'Mira, yo voy a tomar una cerveza, voy al baile', ehh, o hablan como..., a veces. Escupen, el otro día, pasaba y escupía el chicle, así como los hombres. A veces me choca porque la mujer, las jóvenes no deben perder la, digamos, el sentido de ser mujer, la delicadeza ; Verdad? Como que a veces quieren copiar un poco como es el hombre, como es el varón.

Se fracciona el fragmento en tres segmentos que contienen ideas o argumentos distintos entre sí para, posteriormente, analizar el sentido del fragmento seleccionado en su totalidad. El primer segmento iría desde el comienzo de la cita hasta "tenía otra ense-

social spaces they inhabit, and the societies they live in. Analysis of personal narratives can illuminate "individual and collective action and meanings, as well as the social processes by which social life and human relationships are made and changed" (Laslett 1999:392)" (Riessman, 2001:697) 
ñanza" (destacado en negrita). El segundo, por su parte, comienza en "Y que puede tomar la chica" y finaliza en "así como los hombres." A la vez, este segundo segmento podría descomponerse en dos partes, la primera destacada en negrita y cursiva y la segunda sin destacar. Finalmente, el tercer segmento comienza al finalizar el anterior y continúa hasta el final de la cita (destacado en cursiva y subrayado). Con el fin de simplificar las ideas que se expresan en cada segmento, las mismas podrían parafrasearse del siguiente modo:

- S1: Me molesta que los jóvenes se comporten de manera distinta a como yo tuve que comportarme a su edad.

- S2 (primera parte): Hombres y mujeres tienen los mismos derechos.

- S2 (segunda parte): Las mujeres jóvenes quieren alcanzar un derecho que le corresponde al hombre, no a ellas.

— S3: Hay una esencia inherente al "ser mujer" que las jóvenes no están reconociendo.

SEGMENTO 1: "A mí lo que me choca, a mí lo que me choca entre los jóvenes, ya los estoy viendo desde los 13 años, no lo estoy mirando ni siquiera desde los 15,16 , lo que me molesta, porque uno no lo vivió eso, y si tenía ganas no lo podía hacer porque tenía otra enseñanza".

A mí/me: La cita comienza haciendo alusión a que se hablará en primera persona, remarca al sujeto hablante, habla de sí, desde sí mismo. Supone recursos discursivos para empatizar con el grupo y posicionar al habla, al comienzo aparentemente propio, desde una posición compartida por quienes escuchan.

Entre los jóvenes: Introduce quiénes serán objeto del discurso. "Los jóvenes," en tercera persona, señala que existe un "otro" (joven) distinto del sujeto que habla, por contraposición, adulto.

A mí lo que me choca, a mí lo que me choca/lo que me molesta: Se remarca y enfatiza, a partir de la reiteración y posterior énfasis, el hecho de que la realidad que se observa provoca un sentimiento negativo (o muy negativo, dado el énfasis).

Ya los estoy viendo desde los 13 años: La realidad que observa (estoy viendo) remite al "ahora" a partir de la conjugación del verbo "estar" en el presente, acentuada por el uso del "ya." "Ya desde los 13 años" remarca el hecho de que aquello que ve "ahora" ocurre más temprano de lo que esperaría. Quizá aluda a un momento en el tiempo con un doble sentido. Por un lado, a expectativas de comportamiento de las personas según su edad (13 años). Por otro lado, al momento histórico en que se observa.

No lo estoy mirando ni siquiera desde los 15, 16: La referencia "ni siquiera" enfatiza la edad de los sujetos a quién se refiere.

Uno no lo vivió eso: Realiza un giro en el discurso, ya no es "yo" el que "veo" / "vivo" / "hablo," sino "uno." Con este recurso se busca una posición compartida con el grupo, "uno como yo." En el contexto del grupo: "ustedes que también lo vivieron." Si "ya lo vivieron" y ahora no, hay una referencia temporal de la vivencia, es decir, refiere a "ustedes los adultos como yo," que, por contraposición, "ya no son jóvenes." 
Uno ve/uno observa: Lo que observo, lo observa también el grupo.

Porque uno no lo vivió eso: La anticipación de lo que se va a decir con el uso de la palabra "porque" hace alusión a que se van a explicar los motivos que dan origen a aquello a lo que se había hecho referencia con anterioridad ("la molestia" respecto al "otro" o "los jóvenes"). En este sentido, quien habla explica que "le molesta" porque cuando el sujeto tenía la edad de quienes ahora hace referencia "no lo vivió." Posteriormente explica, a la vez, porqué piensa que "no lo vivió."

Porque uno no lo vivó eso, si tenía ganas no lo podía hacer: Al señalar que "si tenía ganas no lo podía hacer" profundiza en la explicación del origen del sentimiento negativo ("la molestia") referida anteriormente. En esta frase expresa una situación hipotética "si hubiese tenido ganas no lo hubiese podido hacer" (imposibilidad de su propia acción). De este modo, la "molestia," explica, no está dada por el comportamiento de las personas a quienes se refiere ("los jóvenes"), sino al hecho de que "los otros" pueden decidir tomar una acción que el sujeto que habla "no hubiese podido," en caso de haberlo deseado. Expresado de otro modo, o parafraseando, la "molestia" radica en la imposibilidad de decisión sobre su propia acción.

Tenía otra enseñanza: Tenía otro sentido de acción, otra interpelación identitaria. Y con esto hace referencia a que los límites provenían de "afuera" aunque fueran internalizados. Lo cual implica que esos límites (dados por las "enseñanzas") no estarían operando con la misma fuerza para los jóvenes que para los adultos (posición del hablante). Esto supone que la mayor "libertad" que le reconoce de hecho a los jóvenes que pueden hacer (por el mero hecho que lo hacen, y eso se puede "ver") no es valorado positivamente en el caso de las performances de género en particular en el caso de las mujeres. Esta idea de la mayor libertad de los jóvenes, menores restricciones que la que tenían los adultos cuando jóvenes, es reiterada en otros grupos de discusión referida a otras dimensiones, que sí son valoradas positivamente.

SEGMENTO 2 (primera parte): "Y que puede tomar la chica, vamo' a decir, somos todos iguales, tenemos los mismos derechos"

Vamo' a decir, somos todos iguales: Desplaza el sujeto emisor del discurso, ya no es un "yo" que habla sino un plural "vamos a decir." El giro discursivo, a partir del uso del plural, permite un cambio en el contenido de aquello que se dice "somos todos iguales" (en el contexto de la conversación: varones y mujeres son iguales). La participante expresa "supongamos que voy a decir," dado que en realidad no es lo que estoy diciendo ("pero yo me opongo" dice a continuación, volviendo a la primera persona singular). Lo que emerge aquí es aquello que se identifica como un "nuevo sentido común," aquella "enseñanza que no tenía," como señaló antes.

Y que puede tomar la chica (...) si fuera un hombre: posibilidad de acción/ señala una posibilidad / sentido ético de autorización de la acción. Sin embargo, la continuación de la frase con el condicional "si" indica que la acción (tomar) solo podría tener lugar bajo la condición de "ser hombre," condición de la cual carece "la chica" y, por tanto, al señalar su posibilidad la desautoriza. 
SEGMENTO 2 (segunda parte): "Pero yo me opongo que una chica, una niña, una chiquilina, una adolescente, ande con una botella como si fuera un hombre. Entonces, a veces eso me choca un poco la cabeza, como que las mujeres, digamos, las niñas o las jóvenes quieren comparase un poco con el mismo derecho del hombre. 'Mira, yo voy a tomar una cerveza, voy al baile, o hablan como..., a veces. Escupen, el otro día, pasaba y escupía el chicle, así como los hombres."

Una chica, una niña, una chiquilina, una adolescente: Se trata de una reiteración que reafirma el sujeto de discurso (jóvenes), a la vez que le asigna una identidad genérica (mujeres jóvenes).

Andan con una botella/ escupen como los hombres: Se reiteran conceptos, acciones que conforman parte del universo simbólico, aquellas representaciones sociales que se interpretan como propias de una identidad genérica de "hombre."

'Mirá, yo voy a tomar una cerveza, voy al baile': El sujeto que se observa (y sobre quien se habla) se dirige a la participante. Señala que no solamente se observa al "otro," sino que se observa porque el otro pide ser observado, "Mirá," interpreta que dice con su acción.

Y que puede tomar la chica (...) pero yo me opongo que (...) ande con una botella como si fuera un hombre: Se señala que aquello que ocasiona la "molestia" no es la acción, sino el modo o lugar en que la acción se realiza (espacio público) o el hecho de que contradiga aquellas representaciones interpretadas como propias de los cuerpos con identidades genéricas asignadas.

Quieren comparase un poco con el mismo derecho del hombre: El derecho, entendido como posibilidad de acción, es, también entendido (como) genérico.

SEGMENTO 3: "A veces me choca porque la mujer, las jóvenes no deben perder la, digamos, el sentido de ser mujer, la delicadeza, ¿Verdad? Como que a veces quieren copiar un poco como es el hombre, como es el varón".

No deben perder: Se señala un sentido ético en la frase al utilizar la palabra "deber", si algo "es" no se puede "perder."

¿Verdad?: Se utiliza un recurso de búsqueda de complicidad del otro, que no espera respuesta.

El sentido de ser mujer, la delicadeza: Se describen las características que posee, en sí, de modo esencial, la mujer biológica.

Quieren copiar un poco como es el hombre: Moverse del esencialismo implica una "actuación", una performance que, en tanto se analiza como tal, pierde sentido de realidad "copiar al hombre" En otras palabras, no puede entender aquellos que queda por fuera esencialismo sino como copia, la imitación, la actuación. Revela el sentido performativo del género. 


\section{Líneas derivadas}

\section{a. Espacios de "publicación"}

Los participantes de los grupos de discusión suelen tener una mirada "laxa" de los espacios públicos de la ciudad para referir a lugares como "la calle," "la vereda," "el ómnibus", "la plaza". Estos espacios se asocian a momentos de encuentro con otros cercanos o extraños. Se caracterizan por ser encuentros efímeros, casuales con desconocidos (Filardo et al, 2005). El término "público" alude, en estos espacios, a la idea de "libre acceso" y también, fundamentalmente, a la de "publicación," es decir, a la presentación de identidades (Filardo, 2012). Se trata del espacio en los que "nos mostramos," donde el otro se "muestra" y se puede hablar de él, donde se lo puede observar, criticar, celebrar, etc.

En el caso de la cita analizada, quien se observa, no solo realiza una acción, sino que pide a su interlocutor ser observado "Mirá, yo voy a tomar una cerveza," dice el "otro" sin hablar de modo explícito. El "otro" "habla" con su comportamiento por habitar un espacio en que se lo puede observar ("la calle," en el caso de referencia).

Ahora bien, en el "espacio público" no se interactúa solamente con "otros," sino con "otros significados" (Filardo et. al. 2005; Filardo et. al. 2008). De acuerdo a Soto (2007):

(...) es en el espacio donde se actualizan y ponen en juego las nociones culturales de género, que se concretan en actividades, prácticas y conductas realizadas cotidianamente, que están estrechamente ligadas con una concepción del mundo y con la construcción subjetiva del sujeto. El género entonces se erigirá como elemento relevante en la producción de imaginarios geográficos imbuidos de simbolismo, poder y significados que dividen esferas, dominios y ámbitos diferenciados. (Soto, 2011: 88)

\section{b. Copia y realidad}

El segundo y tercer segmento de la cita referenciada ameritan algunas reflexiones en torno al modo en que la emisora decodifica las identidades genéricas en los espacios públicos urbanos. En este sentido, el discurso de la participante evidencia la permanencia de una ideología de género usualmente denominada sistema sexo-género (Rubin, 1975).

En el espacio público la interacción se da con "otros significados" (Filardo et. al. 2008). De este modo, aquello se observa, estas identidades presentadas, son interpretadas en base a aquellas representaciones o estructuras normativas sobre el género que el observador pone en práctica. A modo de ejemplo, el comportamiento de las "mujeres jóvenes en la calle" (sujeto del discurso) es interpretado con base en aquellas representaciones que la emisora realiza respecto a qué implica "ser hombre" o "ser mujer" y cómo, en función de estas identidades, existen expectativas respecto a los repertorios de acción identificados como propios de cada cuerpo sexuado. El segundo segmento de la cita de referencia, la participante introduce algunos de los repertorios de acción que interpreta 
como propios, como "derechos inherentes," de cuerpos que performan identidades masculinas en los espacios públicos: beber alcohol, escupir.

“(...) las jóvenes quieren comparase un poco con el mismo derecho del hombre," expresa la participante del grupo de discusión. El "derecho" aquí es interpretado como una posibilidad de acción. Si beber alcohol o escupir es una posibilidad de acción solo identificada como posible en el repertorio masculino, por contraposición, dado que el sistema sexo-genérico construye las categorías dicotómicas varón-mujer como excluyentes, no es una acción posible de ser performada por un cuerpo identificado como femenino.

Ahora bien, ¿Cómo interpretar el hecho de que estos repertorios de acción sean entendidos como inherentes a una determinada identidad genérica? "El sentido de ser mujer," lo llama la participante del grupo de discusión. "La mujer no nace, se hace" declaraba Simone de Beauvoir (2005), a quien cita Judith Butler (2007) para explicar su apropiación de los modelos teatrales y reinterpretarlos. Las ideas de Goffman (2001) sobre la existencia de una performance en la que los sujetos utilizan libretos ya ensayados que requieren una continua confirmación son retomadas por Butler. El género es, en este sentido, un acto que ya ha sido ensayado pero que requiere de actores individuales para ser reproducido una vez más como realidad. "Es preciso discernir los complejos componentes que conforman el acto para entender la suerte de actuación en concierto y de actuación en acuerdo que, invariablemente, es actuar el propio género." (Butler 1990: 306-307) En este sentido, Butler entenderá, en oposición a los modelos fenomenológicos que asumen la existencia de un 'yo' necesariamente antepuesto a sus actos, que los actos constitutivos, además de construir la identidad del actor, la constituyen como una ilusión, como objeto de una creencia.

De este modo, un movimiento, una acción o un repertorio que niega "el sentido de ser mujer" como esencia, no puede interpretarse por fuera de la "copia." La actuación congruente con las normativas de género aparece como la "realidad con sentido," y aquello que se distancia, que se desvía de los esperado, se observa como una copia, una imitación, una actuación y, revela de este modo, el sentido performativo del género señalado por Butler. Esta esencia de la identidad pasa a ser utilizada como técnica en que se sustenta el poder de los discursos normativos sobre la sexualidad. Aquello que se pretende como análisis y descripción que pre-existe al cuerpo, es el propio acto discursivo de producción del dato que pasa a funcionar como su propio mecanismo de legitimación.

\section{c. Indignación y conflicto}

La teoría de la interpelación ideológica de Althusser (2011) sirve a Butler de marco para conceptualizar la formación del sujeto sexuado. Para Althusser, toda ideología ha funcionado constituyendo individuos concretos en sujetos, mediante el mecanismo de la interpelación, definido como el acto de reconocimiento por el cual el sujeto se identifica con aquello con lo que es llamado a identificarse. Así, se sitúa en el lugar que se le ha asignado y asume las prácticas y significados sociales de dicha posición. Por otra parte, el desconocimiento de dicho mecanismo ideológico hace del sujeto aquello con lo que 
se identifica y que pasa a considerar como lo que "desde siempre había sido." (Althusser 2011) Se trata de una operación ideológica de constitución de los sujetos la que genera la "ilusión" de un sujeto esencial anterior a lo social y constituyente del mismo.

"Es en este sentido en el que Butler va a proponer una lectura del sexo como efecto del proceso de naturalización de la estructura social del género y la matriz heterosexual. El sujeto es llamado a identificarse con una determinada identidad sexual y de género sobre la base de una ilusión de que esa identidad responde a una interioridad que estuvo allí antes del acto de interpelación." (Córdoba 2003: 3)

La constitución de la esencia "ilusoria" natural de una identidad, o sujeto como propone Althusser, se desarrolla a través de las propias actuaciones en su repetición compulsiva. Esta idea permite retomar el concepto de performatividad entendida como el poder reiterativo del discurso para producir fenómenos que regula e impone. A través de este concepto se entiende cómo, lo que era considerado un rasgo "interno" de la persona, es algo anticipado, producido a través de ciertos actos corporales, una suerte de "efecto alucinatorio" de gestos naturalizados. "La distinción entre expresión y performatividad es absolutamente crucial, porque si los atributos y los actos del género o sea las diversas maneras en que un cuerpo muestra o produce una significación cultural, son performativos, entonces no hay performatividad pre-existente que pueda ser la vara de la medición de un acto o atributo; no hay actos de género que sean verdaderos o falsos, reales o distorsionados, y el postulado de una verdadera identidad de género se revela como una ficción regulativa." (Butler 1990: 310)

"El sentido de ser mujer, la delicadeza" al que hace alusión la participante revela aquel conjunto de actos corporales, gestos naturalizados con el que ha sido interpelada, revela su propia ficción regulativa del género, su identidad, aquello con lo que ha sido llamado a identificarse. Al afirmar que "si tenía ganas no lo podía hacer porque tenía otra enseñanza" expresa su imposibilidad de acción fuera de la normativa genérica hegemónica al momento de su socialización.

¿Cuál es, entonces, el sentido de la indignación de la participante? La identificación de una mujer biológica que se comporte distinto al "sentido de ser mujer" abre posibilidades de acciones no previstas como repertorio posible en el marco de las normativas del género y, de este modo, revela su propia ficción regulativa, interpela su propia construcción identitaria, aquella percepción de interioridad que, de modo "ilusorio" estuvo allí antes del acto de interpelación.

\section{d. Conflicto entre "clases de edad" y conflicto "generacional"}

En el habla, de acuerdo a Canales (2013), es posible identificar una reproducción de conjunto de normas, modelos, o códigos en base a los cuales el acto es enunciable y comprensible. "Los grupos no hablan, pero nadie puede hablar fuera de las lenguas de sus gru- 
pos" (2013: 184). En este sentido, cuando la participante habla, lo hace, necesariamente, en base a la palabra grupal, es decir, con el "pre-conocimiento" de distinciones, suposiciones y valoraciones que supone, el grupo entiende. En este marco, en el fragmento referido, la participante recurre con frecuencia a la utilización de la primera persona, a la vez que recurrentemente utiliza el sujeto "uno" en base al cual se desplaza quien enuncia, para hacer alusión al grupo, "uno como yo," es decir, quienes nos encontramos conversando: "nosotros los adultos", quienes pertenecemos a la misma época, somos contemporáneos.

Quien enuncia el discurso es, por lo tanto, un adulto que encuentra entre los participantes del grupo un interlocutor semejante, establece un "nosotros" y, en este marco, comienza a hablar de un "otro". "Los jóvenes," en este marco se convierten en el objeto de discurso; ellos pertenecen a una "misma época", de la cual "nosotros" no formamos parte.

La "indignación", en tanto emoción que se expresa en el discurso, no solo pone en evidencia el carácter performativo del género sino que también, manifiesta conflictos entre generaciones (cohortes), que en este marco, se expresan como de clases de edad. La idea de 'clase de edad' remite a la categorización que se establece, en el seno de cada grupo a partir del trazado de fronteras entre distintas condiciones asociadas a la edad -'joven', 'adulto', 'viejo'...-, cada una con una serie de derechos, obligaciones, comportamientos (Martín Criado, 2009). Así, quienes se indignan son los "adultos" en relación al comportamiento de las "jóvenes". El conflicto se expresa utilizando las clases de edad como criterio clasificatorio de los sujetos protagonistas: jóvenes-adultas, a la vez que el discurso asigna a los primeros repertorios de comportamiento y desautoriza líneas de acción con base en la edad.

Ahora bien, el conflicto trasciende estas disputas y adquiere características propias de un conflicto "generacional." La idea de generación alude a las diferencias entre los miembros de diferentes cohortes de un grupo social "cuando cambian las condiciones materiales y sociales de existencia y de reproducción de ese grupo" (Martín Criado 2009, 1). En este sentido, la utilización del concepto conlleva la necesidad de comprender las "condiciones materiales y sociales de existencia" en que se produce el acto de la interpelación. "Tenía otra enseñanza”, manifiesta la participante para aludir a su contexto de socialización, contexto común para quienes nacieron en una misma época, una cohorte de nacimiento compartido en el grupo. La posición grupal evidencia la existencia de "clases de biografías orquestadas y sometidas a un ritmo según el mismo tempo." (Bourdieu, 2002:465)

\section{Reflexiones finales}

En las últimas décadas se ha observado, tanto en Uruguay como en otros países de América Latina, una explosiva emergencia de debates sobre las relaciones de género y la sexualidad que colocan en la discusión pública temas relacionados a numerosas instituciones sociales contemporáneas como el Estado, la educación, la medicina o la cultura popular. Emergen, en este marco, demandas como el aborto, la educación sexual o el matrimonio de personas del mismo sexo que se colocan como foco de atención para los medios 
de comunicación y adquieren una mayor relevancia en las agendas de los gobiernos y las campañas políticas.

Ahora bien, los discursos analizados en el presente trabajo ponen en evidencia la importancia de entender cómo las normativas sobre la sexualidad y los discursos que las sustentan, no son de carácter universal. Por el contrario, conviven en un mismo contexto socio-histórico rasgos de ideologías conservadores con otros de carácter innovador en un entramado complejo de prácticas y relaciones sociales de reproducción, pero también de cambio. He aquí la importancia de colocar el foco en los lugares opacos, en las tensiones y conflictos que esto supone. ¿Cómo se delimitan las prohibiciones y los espacios de trasgresión? ¿Para quiénes, dónde y cuándo se habilitan (o no) determinados repertorios de acción? ¿Qué discursos los sustentan? ¿Quiénes son sus emisores/as y quiénes su objeto?

Del análisis del fragmento seleccionado surge la tensión que viven particularmente las mujeres adultas en torno a la aceptación de las premisas de la igualdad de género y el reconocimiento de las diferencias entre varones y mujeres (que han formado parte de su socialización y que han incorporado). El espacio público es el "locus" idóneo en que esta tensión emerge: al observar cómo bajo la consigna de la igualdad, la performance genérica de las mujeres jóvenes, hace que se comporten "como si" fueran varones, desde la perspectiva de las adultas. Emerge así, la indignación de las adultas, como respuesta a estos comportamientos observables, "publicados" (hechos públicos) de las mujeres jóvenes.

El discurso es enunciado en contexto situacional en que se habla para otros que pertenecen a la misma generación, dando por supuesto que lo dicho hace sentido (Martín Criado, 2014). Se identifica una representación "otra" a partir de la cual se dialoga y, aún más, a la cual, en términos formales, se adscribe: "somos todos iguales, 'vamo a decir." No se expresa una intencionalidad explícita de contra-argumentación, sino, más bien, de reconocimiento de igualdad entre varones y mujeres y, aun así, se la interpela.

Se destaca que el objeto de discurso sólo remite a las mujeres y no hace alusión alguna a los comportamientos masculinos, salvo como referente de imitación.

Se trata de un conflicto especificado en las mujeres de diferentes generaciones, las que habitan este contexto socio-histórico: las jóvenes, que incorporan (hacen cuerpo, en sus propios cuerpos) los cambios, y las adultas o adultas mayores, que habiendo sido socializadas en la "esencia de lo femenino" no lo encuentran en sus congéneres de menor edad y se indignan por ello. Quizá sea oportuno distinguir que no es un conflicto de clase de edad (adultos-jóvenes) sino que se expresa a partir de la edad, en estas generaciones que comparten este momento histórico en el que se rompe la reproducción del mandato de género y modifica así la performance de ser mujer en el espacio público. Mientras el sentido del ser mujer era transmitido de generación en generación conservando su performance genérica (representando en el espacio público lo que se entendía "debía hacer una mujer") no se manifiesta este conflicto. Emerge cuando se quiebra el mandato. Sin embargo, en el discurso de los sujetos analizados se utilizan las clases de edad como criterio clasificatorio de los sujetos protagonistas (jóvenes- adultas).

El tiempo social y el tiempo individual se condensan en el conflicto analizado para las generaciones de mujeres adultas y adultas mayores (cohortes de nacidas antes de 1970). 
Viven hoy un momento histórico de consagración normativa y discursiva de la equidad de género, que si bien formalmente acompañan, entra en tensión con el mandato genérico de ser mujer en que se socializaron en el momento histórico en que fueron jóvenes. La tensión se manifiesta en el locus del espacio público urbano al observar la performatividad de género de las mujeres jóvenes que las interpela.

\section{Bibliografía}

ALTHUSSER, LOUIS. 2011. “Ideology interpellates individuals as subjects” en Gay, D, Evans, J. y Redman, P. (Ed.) Identity: a reader, Londres, SAGE, pp. 31-38.

ARENDT, HANNAH. 1997. ¿Qué es política? Barcelona, Ediciones Paidós.

BOURDIEU, PIERRE (2002) La distinción. Criterios y bases sociales del gusto. Taurus. México. BUTLER, JUDITH. 1990. “Actos performativos y constitución del género.” Case, Ellen (Ed.) Performing Feminisms: Fememinist Critlical Theory and Theatre, University Press, pp. 720-728.

- 2007 [1999]. "El género en disputa”. Paidós, Barcelona.

BRUNER, J. (1991). "The narrative construction of reality". Critical Inquiry. 18(1): 1-21.

CANALES, MANUEL., 2013. "Análisis sociológico del habla”. En Canales, Manuel (coord.)

Escucha de la escucha: análisis e interpretación en la investigación cualitativa. Santiago de Chile, LOM ediciones, pp. 171 - 178.

CÓRDOBA, DAVID. 2003. Identidad y performatividad. Barcelona, Athenea Digital.

DE BEAUVOIR, SIMONE. 2005 [1949]. “El segundo sexo”. Cátedra, España.

DEL VALLE, TERESA. 2006. "Procesos de la memoria. Cronotopos genéricos" En Revista

Virtual La Ventana, №9, pp 211-225. Centro Universitario de Ciencias Sociales de la Universidad de Guadalajara, México

FILARDO, VERÓNICA, AGUIAR, SEBASTIÁN, CARDEILLAC, JOAQUÍN, FARÍAS, EMA, NOBOA, LAURA (2005). La ciudad: las múltiples ciudades. Documento de Trabajo №

73. Departamento de Sociología - Universidad de la República. Montevideo

FILARDO, VERÓNICA, MUÑOZ, CARLOS, AGUIAR, SEBASTIÁN, CHOUHY, GABRIEL, NOBOA, LAURA, ROJIDO, EMILIANO, SCHINCA, PEDRO (2008) Presentación de resultados del proyecto: Usos y apropiaciones de espacios públicos de Montevideo y clases de edad. Montevideo, DS-FSC-UdelaR.

FILARDO, VERÓNICA. 2012. "Miedos urbanos y espacios públicos en Montevideo" Disponible en: http://www.geug.edu.uy/wp-content/uploads/2012/08/miedos-urbanos-yespacios-p\%C3\%BAblicos-filardo.pdf Consultado el 25 de Julio de 2017

FILARDO, V. (2018) El tiempo pasa. Marcos de referencia y marcas generacionales. En El Uruguay desde la Sociología № 16. Departamento de Sociología Facultad de Ciencias Sociales. pp 243-258

FILARDO, V. (2020) Narrativas de la Pérdida. (en revisión)

GOFFMAN, ERVING. 2001. "La presentación de la persona en la vida cotidiana." Amorrortu, Buenos Aires. 
LIND, AMY y ARGÜELLO, SOFÍA (2009) “Ciudadanías y Sexualidades en América Latina. Presentación del dossier" Íconos. Revista de Ciencias Sociales 35: 13-18. Facultad Latinoamericana de Ciencias Sociales-Sede Académica de Ecuador. [ISSN: 1390-1249]

MARTÍN CRIADO, E. (2009) Clases de edad. Generaciones. En Reyes, Román (dir) Diccionario Crítico de Ciencias Sociales. Disponible en https://webs.ucm.es/info/eurotheo/ diccionario/C/index.html. Consultado el 30 de julio de 2018.

ORTNER, SHERRY. 1979. “¿Es la mujer con respecto al hombre lo que la naturaleza con respecto a la cultura?” En Young, Kate y Harris, Olivia (comp.) Antropología y feminismo. Ediciones Anagrama, Madrid

POLKINGHORNE, D (1998) Narrative Knowing and the Human Sciences. State University of New York Press. USA.

RIESSMAN, C. (2001) Analysis of personal narratives in Gubrium, J.F. \& J.A. Holstein, Handbook of Interviewing, Sage Publications, 2001.

ROSALDO, MICHELLE. 1979. “Mujer, cultura y sociedad: una visión teorica.” En Young, Kate y Harris, Olivia (comp.) Antropología y feminismo. Madrid, Ediciones Anagrama.

RUBIN, GAYLE. 1975 "The traffic in women: notes on the political economy of sex" En Reiter, R. (ed.). Toward and Anthropology of Women. New York, Monthly Review Press, pp. 157-210.

SIG-INMUJERES. 2016. "Construcciones de la masculinidad hegemónica: una aproximación a su expresión en cifras." Cuadernos del Sistema de Información de Género, Inmujeres-MIDES.

SOTO, PAULA. 2003. “Sobre género y espacio: una aproximación teórica.” Disponible en: http://bvirtual.ucol.mx/ Consultado el 29 de marzo de 2017.

- (2007) “Ciudad, ciudadanía y género. Problemas y paradojas” En Territorios, pp 29-46. Bogotá, Universidad del Rosario, pp. 29-45. 